\title{
Dispersive mudslide-induced tsunamis
}

\author{
A. Rubino ${ }^{1}$, S. Pierini ${ }^{2}$ and J. O. Backhaus ${ }^{1}$ \\ ${ }^{1}$ Institut für Meereskunde. Universität Hamburg, Troplowitzstraße 7, D-22529 Hamburg, Germany \\ 2Dipartimento di Fisica. Università dell'Aquila. Via Vetoio. I-67010 Coppito (l'Aquila), Italy
}

Received: 27 May 1997 - Accepted: 15 May 1998

\begin{abstract}
A nonlinear nested model for mudslide-induced tsunamis is proposed in which three phases of the life of the wave, i.e. the generation, far-field propagation and costal run-up are described by means of different mathematical models, that arc coupled through appropriate matching procedures. The generation and run-up dynamics are simulated through a nonlinear shallow-water model with movable lateral boundaries: in the generation region two active layers are present, the lower one describing the slide descending on a sloping topography. For the intermediate phase, representing wave propagation far from the generation region, the hydrostatic assumption is not assumed as appropriate in general and, therefore, a nonlinear model allowing for weak phase dispersion, namely a Kadomtsev-Petviashvili equation, is used. This choice is made in order to assess the relevance of dispersive features such as solitary waves and dispersive tails. It is shown that in some realistic circumstances dispersive mudslide-induced tsunami waves can be produced over relatively short distances. In such cases the use of a hydrostatic model throughout the whole tsunami history turns out to give erroneous results. In particular, when solitary waves are generated during the tsunami propagation in the open sea, the resulting run-up process yields peculiat wave forms leading to amplified coastal inundations witt respect to a mere hydrostatic context.
\end{abstract}

\section{Introduction}

There is a number of different generating mechanisms for tsunami waves (Sabatier, 1986). The most common mechanism of tsunami generation is related to seismic events, for which the impulsive movement of the water column produced by the displacement of the ocean bottom lcads to an almost instantaneous formation of a surface disturbance (Hammack, 1973). The sudden movement of a mudslide over an incline due to various geological reasons can also lead to the formation of a surface disturbance. Slide-induced tsunamis are usually more localized than seismic ones, but can nonetheless be responsible of severe damages, especially when the wave is trapped over narrow topographic features, which prevent energy radiation of the disturbance (Murty, 1979). It is to be stressed that the relation between slide phenomena and seismic activity, both being agent of tsunami generation, can explain the apparent mismatch between earthquake intensity and tsunami response (Satake et al., 1993, Kanamori and Kikuchi, 1993).

Unlike most seismic tsunamis, for slide-induced tsunamis the surface disturbance is generated by the creeping motion of a lower boundary. In this case the slide motion and the corresponding surface elevation are coupled, the latter depending on the history of the bottom movement. On the other hand the motion of the slide itself is influenced by the presence of the generated tsunami. This two-way coupled system was first investigated by Jiang and Le Blond (1992, 1993) for the case of a slide-tsunami system on a simple wwo-dimensional sloping bottom. More recently the threedimensional case was considered (Jiang and Le Blond, 1994). Rulbino (1994) studied a similar problem and discussed an application to realistic topographies. All these resutis show a complex evolution of such a system. The strface manifestation of the slide motion consists of a crest and a trough propagating toward the open ocean, of a forced depression travelling with the same velocity of the slide and of a trough followed by a crest propagating onshore. The slide undergoes continuous changes in its shape and develops an ondulatory tail, the action of the surface wave on the slide being of secondary importance.

In all these studies, focused on the generating phase of the tsunami, the hydrostatic assumption is made, which -as a consequence- leads to non-dispersive wave evolution. It can in fact be shown that there exists an intermediate far-field 
extending from the hypocenter of the phenomenon to a certain distance (being function of the water depth and of the spatial extension of the bottom movement), for which dispersive effects are negligible (Tuck and Hwang, 1972). While the hydrostatic approximation is in most cases quite acceptable for modelling the generation process, in the successive far-field propagation phase dispersive effects should in general be taken into account since their combined effect with nonlinearity would (if the wave can travel for a sufficiently long distance) lead eventually to solitary vave production (e.g. Hammack, 1973, Hammack and Segur, 1974). In fact, while in large open oceans solitary waves are commonly produced by energetic seismic tsunamis, the life of a tsunami generated near the coast may be not long enough for dispersive effects to become important.

In this paper a process study is presented showing that, nevertheless, coastal tsunamis generated by slide movements can evolve into a train of rank-ordered solitary waves in some realistic circumstances, so that neglecting dispersive effects in modelling wave propagation can lead in some realistic cases- to erroncous results. A nonlinear nested model for mudslide-induced tsunamis is presented in which three phases of the life of the wave, i.e. the generation, the far-field propagation and the costal run-up are described by means of different mathematical models (Sect. 2), that are coupled through appropriate matching procedures (Sect. 3). The generation and run-up dynamics are simulated through a shallow-water model with movable lateral boundarics (Rubino, 1994). In the generation region two active layers are present, the lower one describing the slide descending on a sloping topography.

For the intermediate phase, representing wave propagation far from the generation region, the hydrostatic assumption is not assumed as appropriate in general and a threedimensional model allowing for weak phase dispersion, namcly a regularized Kadomtsev-Petviashvili equation (Kadomtsev and Petviashvili, 1970, Pierini, 1986, 1989) is applied to model tsunami propagation (Sect. 4). This choice is made in order to assess the relevance that dispersive features such as solitary waves and dispersive tails can have in realistic situations. It is shown that for initial slides with geometrical characteristics typical of coastal regions, dispersive waves can be produced over relatively short distances. In such cases a merely hydrostatic description of tsunami evolution would lead to erroneous results. In particular, when solitary waves are generated during tsunami propagation, the resulting run-up process yields peculiar wave forms leading to amplified coastal inundations with respect to a mere hydrostatic context.

Finally, in Sect. 5 sensitivity experiments relative to initial slides with different volumes are carried out in order to asses their importance in wave cvolution, and the role played by a fjord in inhibiting the horizontal energy spreading and therefore in modifying the following wave evolution is investigated.

\section{The generation, the far-field propagation and the run- up models}

The dynamics of the mudslide, of the surface waves which it generates and of the interaction processes occurring between mudslide and surface waves are simulated by mcans of a two-layer model (Rubino, 1994). The model is based on the nonlinear, hydrostatic, shallow water equations which include horizontal momentum diffusion as well as interface and bottom friction terms. In the following we denote the vertically avcraged velocity and transport vectors by $\boldsymbol{u}_{i}$ and $\boldsymbol{U}_{i}=\boldsymbol{u}_{i} h_{i}(i=1,2)$ respectively. The subscripts $i=$ 1 and $i=2$ refer to the upper and lower layer respectively. For the upper layer, the momentum and the continuity equations read:

$$
\begin{aligned}
& \frac{\partial \mathbf{U}_{1}}{\partial t}+\nabla_{h} \bullet\left(\mathbf{u}_{1} \otimes \mathbf{U}_{1}\right)=-g h_{1} \nabla_{h} \zeta- \\
& \frac{\kappa_{1}}{\rho_{1}}\left(\mathbf{u}_{1}-\mathbf{u}_{2}\right)\left|\mathbf{u}_{1}-\mathbf{u}_{2}\right|+A_{h} \nabla_{h}^{2} \mathbf{U}_{1}, \\
& \frac{\partial h_{1}}{\partial t}+\nabla_{h} \cdot \mathbf{U}_{1}=0
\end{aligned}
$$

while for the lower layer they read:

$$
\begin{aligned}
& \frac{\partial \mathbf{U}_{2}}{\partial t}+\nabla_{h} \bullet\left(\mathbf{u}_{2} \otimes \mathbf{U}_{2}\right)=-g \frac{\rho_{1}}{\rho_{2}} h_{2} \nabla_{h} \zeta-g^{\prime} h_{2} \nabla_{h} \zeta^{\prime}+ \\
& \frac{\kappa_{1}}{\rho_{2}}\left(\mathbf{u}_{1}-\mathbf{u}_{2}\right)\left|\mathbf{u}_{1}-\mathbf{u}_{2}\right|-\frac{\kappa_{2}}{\rho_{2}} \mathbf{u}_{2}\left|\mathbf{u}_{2}\right|+A_{h} \nabla_{h}^{2} \mathbf{U}_{2}, \\
& \frac{\partial h_{2}}{\partial t}+\nabla_{h} \cdot \mathbf{U}_{2}=0,
\end{aligned}
$$

Here $\nabla_{h}$ denotes the horizontal Nabla operator, $\bullet$ the scalar product, and $\otimes$ the tensor product, $h_{1}$ and $h_{2}$ are the thicknesses of the upper and lower layer respectively, $\zeta$ is the sea surface displacement and $-\zeta^{\prime}$ the distance of the interface from the mean sca surface, $\rho_{1}$ and $\rho_{2}$ are the densities of the upper water layer and of the slide respectively, $g^{\prime}=g\left(\rho_{2}-\rho_{1}\right) / \rho_{2}$ is the reduced gravity, where $g$ denotes the acceleration of gravity, $A_{h}$ is the horizontal eddy viscosity coefficient, and $\kappa_{1}$ and $\kappa_{2}$ arc the interface and bottom friction coefficients, respectively. Note that the densities $\rho_{1}$ and $\rho_{2}$, as well as the coefficients $A_{h}$, $\kappa_{1}$, and $\kappa_{2}$ are assumed to be constant.

For the simulation of the dynamics of a localized layer, movable lateral boundary conditions are required (Backhaus, 1976, Jungclaus and Backhaus, 1994, Rubino, 1994). The preceding equations $(1,2)$ are discretized in an Arakawa C-grid and solved by means of an explicit Eulerian algorithm. The advective terms are computed by means of an upstream method.

The hydrostatic model $(1,2)$ does not include phase dispersion. In order to take into account such effect in the far-field evolution of the tsunami wave, a non-hydrostatic, 
nonlinear, regularized Kadomtscv-Petviashvili equation (Picrini, 1986, 1989),

$$
\begin{gathered}
\frac{\partial}{\partial x}\left[\frac{\partial \zeta}{\partial t}+\left(c_{o}+\beta \zeta\right) \frac{\partial \zeta}{\partial x}-\frac{\alpha}{c_{o}} \frac{\partial^{3} \zeta}{\partial x^{2} \partial t}\right]+\frac{c_{o}}{2} \frac{\partial^{2} \zeta}{\partial y^{2}}=0, \\
c_{o}=\sqrt{g D}, \quad \alpha=D^{2} \frac{c_{o}}{6}, \quad \beta=\frac{3}{2} \sqrt{\frac{g}{D}}
\end{gathered}
$$

(where $c_{0}$ is the nondispersive phase speed and D the water depth) is nested with the generation model. Equation (3), which is able to describe the evolution of long, nonlinear, weakly dispersive and weakly three-dimensional surface waves, is solved by means of an implicit, three-time-level finite difference scheme. In all cases (3) is solved in a Galileian reference frame moving with the nondispersive phase specd $c_{0}$. The size of the domain of integration along $\mathrm{x}$ can thus be greatly reduced.

As the wave, approaching an opposed coastal region, encounters decreasing water depth and eventually inundates the beach, the KP dynamics no longer applies. Therefore, in order to describe the final wave run-up process, the wave produced by (3) is taken as the initial condition for the onelayer shallow water model given by $(1,2)$ with $\zeta^{\prime}=-D$. In this model movable lateral boundaries such as those previously applied to the slide are implemented for the surface layer.

In the generation model $(1,2) \Delta x=\Delta y=62.5 \mathrm{~m}$ and $\Delta t=0.5 \mathrm{~s}$, while in the propagation model $\Delta x=62.5 \mathrm{~m}, \Delta y=20 \cdot \Delta x=1250$ $\mathrm{m}$ and $\Delta t=2.82 \mathrm{~s}$. In the final run-up model $\Delta x$ ranges from $125 \mathrm{~m}$ up to $10 \mathrm{~m}$. In $(1,2)$ the density contrast is $\rho_{2} / \rho_{1}=2$, $A_{h}=50 \mathrm{~m}^{2} \mathrm{~s}^{-1}, \kappa_{1}=0.5 \mathrm{~kg} \mathrm{~m}^{-3}$ and $\kappa_{2}=5 \mathrm{~kg} \mathrm{~m}^{-3}$.

\section{The matching procedure}

In this section the generation/far-field propagation and the far-field propagation/run-up matching procedures are descibed in detail. The coupling between the generation and the far-field propagation models described in the preceding section needs a matching which has to satisfy the following requirements: due to the unidirectional nature of (3) any initial condition must be travelling in the positive $\mathrm{x}$ direction, the matching time must be chosen so as to ensure that dispersive effects are not developed yet, the surface structures have to be weakly $3 \mathrm{D}$ and, finally, they should lie on a flat bottom. Once a state has been reached for which these conditions are satisfied for the lcading wave, the latter must be separated from the surface disturbance overlying the slide. The main surface structures produced by the generation model $(1,2)$ after an initial transient phase consist of a leading crest and trough followed by a region of small amplitude surface disturbances and, finally, by a forced wave superimposed on the slide. The initial condition for (3) is thus obtained by imposing $\zeta=0$ in the region of the forced wave. A crude cut-off would however
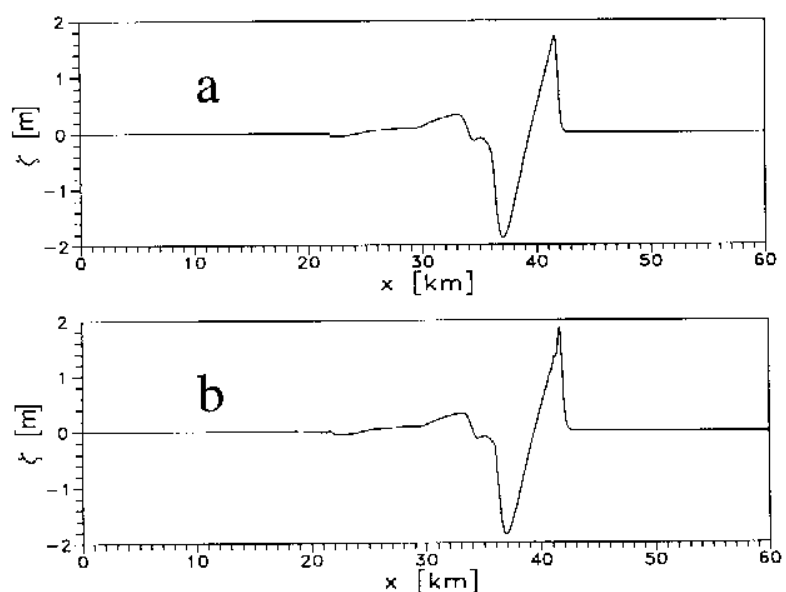

Fig. 1. (a): Wave form at $t=t_{m}=1300 \mathrm{~s}$ after the hydrostatic evolution $(1,2)$. (b): Wave form at $t=1300 \mathrm{~s}$ after the hydrostatic evolution up to $t=1100 \mathrm{~s}$ and then according to Eq. (3) up to $t_{m}$.

introduce artificial gradients in the surface displacement: to avoid this the diffusive recursive algorithm

$\Delta \zeta=q \nabla_{H}^{2} \zeta$

with $q=0.1 \mathrm{~m}^{2}$ is applied in the band of small amplitude disturbances, so as to ensure the smoothness of the transition region.

As observed above, the matching has to be applied before the effects of phase dispersion have become important. To this respect a matching time $t_{m}$ is determined empirically and the non-dispersive cvolution up to that time is considered (as an example see Fig. 1a referring to the most energetic case examined in Sect. 4). To check that $t_{m}$ belongs to a time domain in which phase dispersion is still negligible, the wave is allowed to evolve according to (3) from $t=t_{m}-\Delta t$ to $t_{m}$ (Fig. 1b). The similarity between the two pictures confirms the validity of the choice of $t_{m}$. Such control has been carried out in all the proposed numerical experiments.

Let us now pass to consider the coupling between the farfield propagation model and the run-up model. The matching is performed in the region of flat bottom and consists in prescribing the field produced by the KP equation as the initial condition for the run-up model. Model $(1,2)$ needs the sea surface elevation and the velocities to be prescribed at the matching time, while only $\zeta$ is explicitly computed by means of model (3). Approximate expressions for the velocities have therefore been derived in the following way. The linear, nondispersive and one-dimensional approximation of (3) is given by:

$\frac{\partial \zeta}{\partial t}+c_{o} \frac{\partial \zeta}{\partial x} \equiv 0$ 
On the other hand from the linearized shallow water equations one has:

$$
\frac{\partial u}{\partial t} \cong-g \frac{\partial \zeta}{\partial x}
$$

Combining (5) and (6) yields the velocity component in the direction of propagation:

$$
u \cong \sqrt{\frac{g}{D}} \zeta .
$$

The transversal velocity component can then be obtained by considering the continuity equation,

$$
\frac{\partial \zeta}{\partial t}+D\left(\frac{\partial u}{\partial x}+\frac{\partial v}{\partial y}\right)=0
$$

which, together with (7) gives

$$
\frac{\partial v}{\partial y} \cong-\frac{1}{D} \frac{\partial \zeta}{\partial t}-\sqrt{\frac{g}{D}} \frac{\partial \zeta}{\partial x} .
$$

Equation (8) can finally be integrated with the lateral boundary conditions $\mathrm{v}=0$.

\section{The role of phase dispersion in the far-field propagation}

In all the numerical experiments presented (Table 1) the bottom topography (Fig. 2) represents two opposed coastal areas connected by two slopes and an intermediate deeper region. The slide is initially located over one of the slopes (with an inclination of $3^{\circ}$ ) and its shape consists of an elliptical paraboloid with an exponential head (Fig. 3). The axis of the base of the ellipsoids are $a=2300 \mathrm{~m}$ and $b=1800$

Table 1. List of all the experiments presented. The cvolution in sectors a, $b$ and $d$ (see Fig. 2) is always according to the hydrostatic model $(1,2)$. Over the "far-field shelf" (sector c) the evolution according to the hydrostatic or to the dispersive (3) model is specified.

\begin{tabular}{lll} 
Experiment: & $\begin{array}{l}\text { Initial slide volume } \\
\left(\times 10^{8} \mathrm{~m}^{3}\right)\end{array}$ & Evolution in sector c: \\
I & 3.1 & dispersive \\
II & 3.1 & non-dispersive \\
III & 4.6 & dispersive \\
IV & 0.2 & dispersive \\
V & 0.8 (within fjord) & dispersive \\
VI & 1.3 & dispersive \\
\hline
\end{tabular}

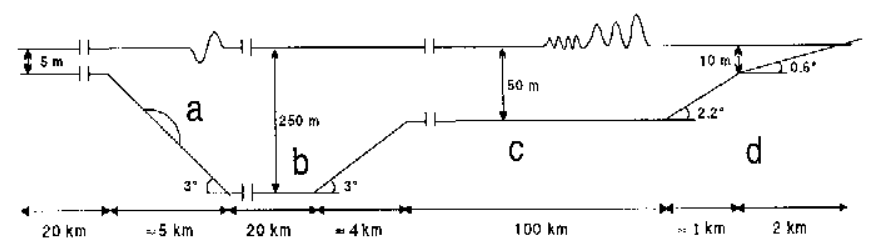

Fig. 2. The bathymetry used in the numerical experiments (not in scale). The sediments are placed in sector a. The surface waves thus generated cvolve according to $(1,2)$ in regions $\mathrm{a}$ and $\mathrm{b}$. At the beginning of sector $\mathrm{c}$ the coupling with the dispersive model (3) is performed for all the experiments except for Exp. II. In sector $d$ the final run-up process is modeled again according to $(1,2)$.

$\mathrm{m}$ and different volumes $\mathrm{V}$ of the slide will be considered by changing the amplitude of the structure. In the final case (Sect. 5) a lateral topographic constriction is added in order to schematize the presence of a fjord.

We stress that it is the very existence of the far-field shelf region (sector $c$ of Fig. 2) that can make dispersive effects important. The propagation over deeper water (sector b) of these low energy tsunamis would hardly exhibit any nonhydrostatic featurc (the length and depth of this sector which we chose as $20 \mathrm{~km}$ and $250 \mathrm{~m}$ respectively- are virtually irrelevant to this respect). This is because the local Ursell number (defined as the ratio between nonlinear and dispersive terms, $\mathrm{Ur}=\zeta \mathrm{L}^{2} / \mathrm{D}^{3}$, where $\mathrm{L}$ is a typical horizontal length scale of the wave, Ursell, 1953) is found to be less than unity in most cases. However, when the wave transits into a shallower region (sector $\mathrm{c}$ ) Ur experiences a sudden increase that leads in many realistic circumstances (see below) to the rapid development of dispersive features.

In the present section, in order to stress the importance of phase dispersion in the far-field propagation, the coupled model will be run in the case of an intermediate slide volume and the resulting wave will be compared to the corresponding one as obtained by the application of the hydrostatic model also in the far field. The importance of phasc dispersion in the far-field propagation for the subsequent run-up will finally be investigated.

The total volume of the slide is $\mathrm{V}=3.1 \times 10^{8} \mathrm{~m}^{3}$ (Exp. I). At the matching time $t_{m}=1300 \mathrm{~s}$ (which turns out to be a correct value for all the proposed experiments), just after the slope

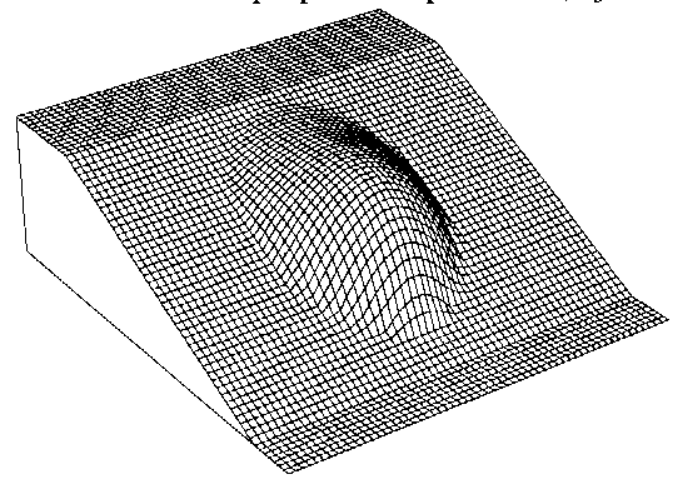

Fig. 3. The initial form of the sediments generating the underwater slide. 
leading to sector $\mathrm{c}$, the leading wave has the form of a steep crest followed by a trough (Fig. 4A). The corresponding Ursell number is $\mathrm{Ur}>\mathrm{O}(10)$. Thus solitons are expected to emerge in the subsequent propagation (note that the mechanism of soliton formation in 3D is basically the same as in 2D, Pierini, 1989). Furthermore the leading crest is very stecp so that the balance between dispersive and nonlinear effects, that are the basis of solitary wave formation, should be reached very soon. More precisely the following formula provides an upper bound for the time required for the first soliton to emerge (Pierini, 1989):

$$
\left\{\begin{array}{l}
T_{s}=\frac{L_{\theta}-L_{s}}{\beta \zeta_{o}} \\
L_{s}=\sqrt{\frac{4 D^{3}}{3 \zeta_{o}}}
\end{array}\right.
$$

where $L_{\circ}$ is the length scale of the leading crest at the matching time, $\zeta_{o}$ the corresponding elevation and $L_{s}$ is the length scale of a $1 \mathrm{D}$ soliton with the amplitude $\zeta_{0}$. From (9) the first soliton is to be expected after $\mathrm{T}_{\mathrm{s}}=\mathrm{O}(10 \mathrm{~min})$ and $\mathrm{L}_{\mathrm{s}}=\mathrm{O}(10 \mathrm{~km})$.

The preceding theoretical considerations are confirmed by the numerical results. Fig. 4B shows the dispersive evolution of the wave form of Fig. 4A, while in Fig. 4C the axial profiles at different times are given. The wave develops into a series of three $3 \mathrm{D}$ solitary waves followed by a strong dispersive tail. Such evolution is typical of a weakly nonlinear and dispersive dynamics (e.g. Hammack and Segur, 1974), therefore the application of a hydrostatic, nondispersive generation model such as $(1,2)$ also to the farfield propagation over sector $\mathrm{c}$ would presumably produce a completely different scenario. In order to verify this, an extended application of $(1,2)$ was carried out (Exp. II). The results are shown in Fig. 5A (compare with Fig. 4B) and Fig. 5B (compare with Fig. 4C). The nondispersive evolution yields a wave whose shape is basically similar to that at the matching time. Moreover the 3D spreading appears to act more efficiently in the absence of solitary features, and this accounts (along with the frictional effects that are absent in the KP equation) for the faster decrease of the amplitude of the leading wave. This could imply that, in the presence of well developed dispersive effects the repercussions on the coast would be larger than those expected in a mere hydrostatic regime.

It was shown above that the waves in the far-field as obtained by including (Fig. 4C) or neglecting (Fig. 5B) phase dispersion are very different. At this stage one can ask what will be the final run-up produced by these two different structures. In order to investigate this, such situations will be taken as the initial conditions for the runup model as described in Sects. 2,3. Such hydrostatic model does not describe the further dispersive evolution but, if dispersive structures are present initially, then their shape will be preserved, though modified by the presence of the topographic slope. The final topography is composed of a first slope of $2.2^{\circ}$ for a length of $1 \mathrm{~km}$ followed by a second slope of $0.6^{\circ}$ which leads to the shore line after $2 \mathrm{~km}$ (Fig. 2).

In Fig. 6A the axial sections of the wave over the sloping topography are shown for Exp. I. The solitonic structures and the dispersive tail are still detectable and, due to the decreasing water depth, the wave front becomes steeper and steeper and the amplitude increases. The subsequent run-up evolution confirms the tendency for the dispersive structures to survive in a hydrostatic context, as it appears clear in Fig. 6B, where the run-up produced by model $(1,2)$ for a field originating from (3) (Exp. I) is presented. This evolution leads to a more effective coastal run-up than that obtained for a non-dispersive development throughout the whole life of the wave (Fig. 6C, Exp. II) because in the first case larger amplitudes are produced when solitons are formed.

\section{Waves generated by different initial conditions}

In this section, in order to assess the relevance of the initial slide volume and of lateral constrictions in wave evolution, two different initial volumes (one larger and another smaller than that analyzed in Sect. 4) are studied, and the presence of an idealized fjord is then considered.

The first case refers to $\mathrm{V}=4.6 \times 10^{8} \mathrm{~m}^{3}$ (Exp. III). In Fig. 7 the wave at the matching time and the further evolution are presented. The Ursell number is only slightly larger than that of Fig. 4 and the situation is basically similar to the corresponding case. The number of emerged solitons is increased of two units and the dispersive tail appears stronger.

The second case refers to $\mathrm{V}=0.2 \times 10^{8} \mathrm{~m}^{3}$ (Exp. IV). Here $\mathrm{Ur}=\mathrm{O}(1)$ and the crest is less steep than in the previous two cases. As a consequence one soliton -after a larger time- is expected to arise. This is indeed what occurs, as shown in Fig. 8. It should be noticed that some evidence of dispersive behavior is detectable in the lateral domain of Fig. 8B. This is a consequence of the three-dimensional nature of the phenomenon: nonlinear effects decrease in intensity from the radial axis to the periphery, thus leading to a larger relative importance of phase-dispersion in such area.

We finally consider the cvolution of a mudslide generated wave in the presence of a lateral boundary, such as a fjord (Fig. 9) which prevents a fully $3 \mathrm{D}$ spreading in the initial stage of wave radiation. In this case $V=0.8 \times 10^{8} \mathrm{~m}^{3}$ (Exp. V). In Fig. 10 the wave at the matching time and the further evolution are shown. Despite the small initial volume involved, a solitonic development can be noticed in the early stage of the far-ficld. 

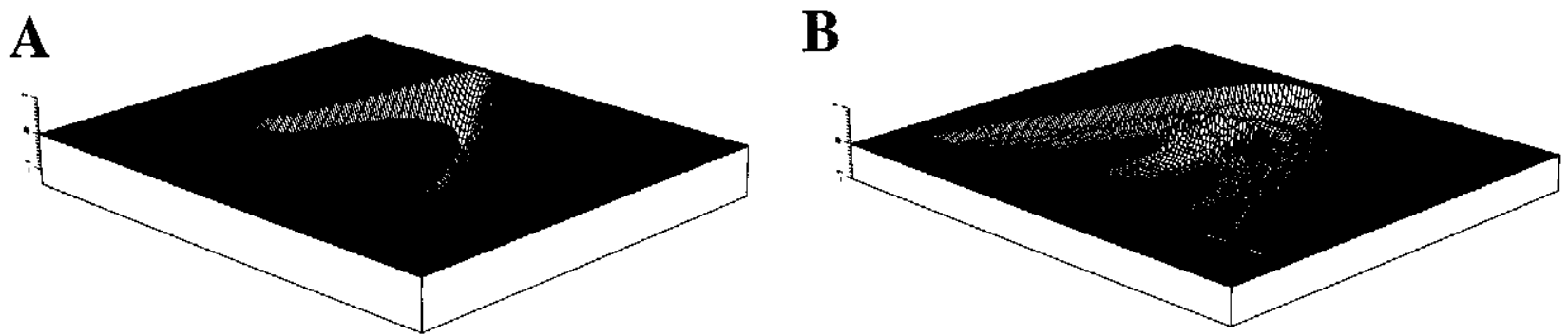

C
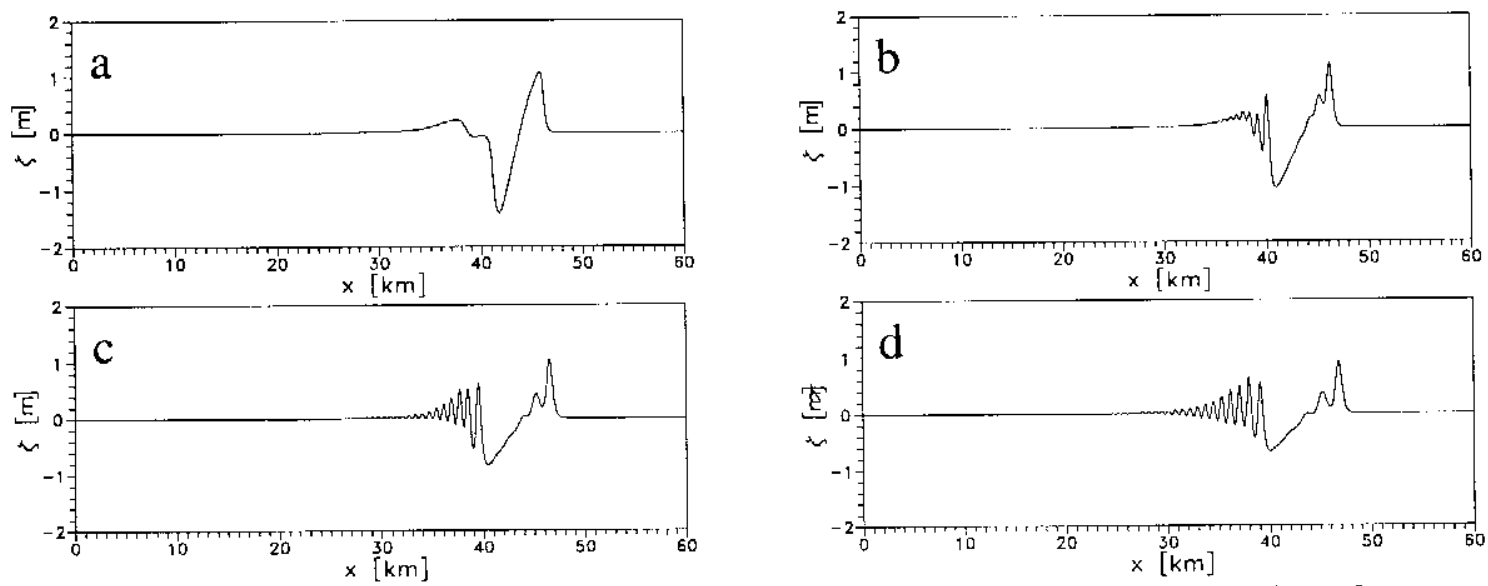

Fig 4. A: Form of the tsunami for experiment $\mathrm{I}$ at $t=t_{m}$. The wave is located at the beginning of sector c. B: Tsunami evolution for Exp. I, $92 \mathrm{~min}$ after the coupling with the non-hydrostatic model (3). The wave is still located in sector $\mathrm{c}$. C: Axial sections of the tsunami for Exp. I at the matching time $t_{m}$ (a) and after 23 (b), 46 (c) and 69 (d) min. In all cases the wave is located in sector $\mathrm{c}$.

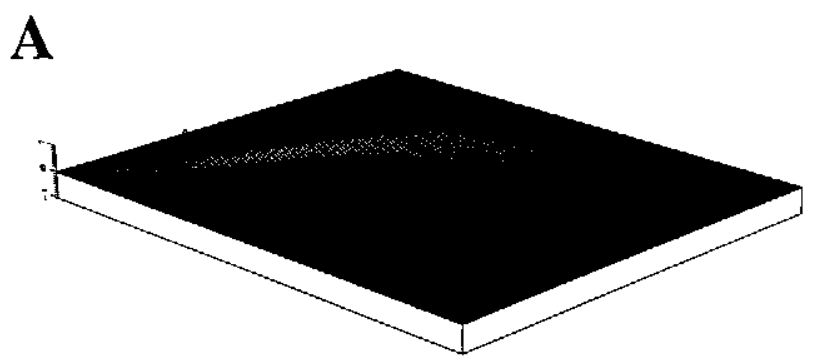

B
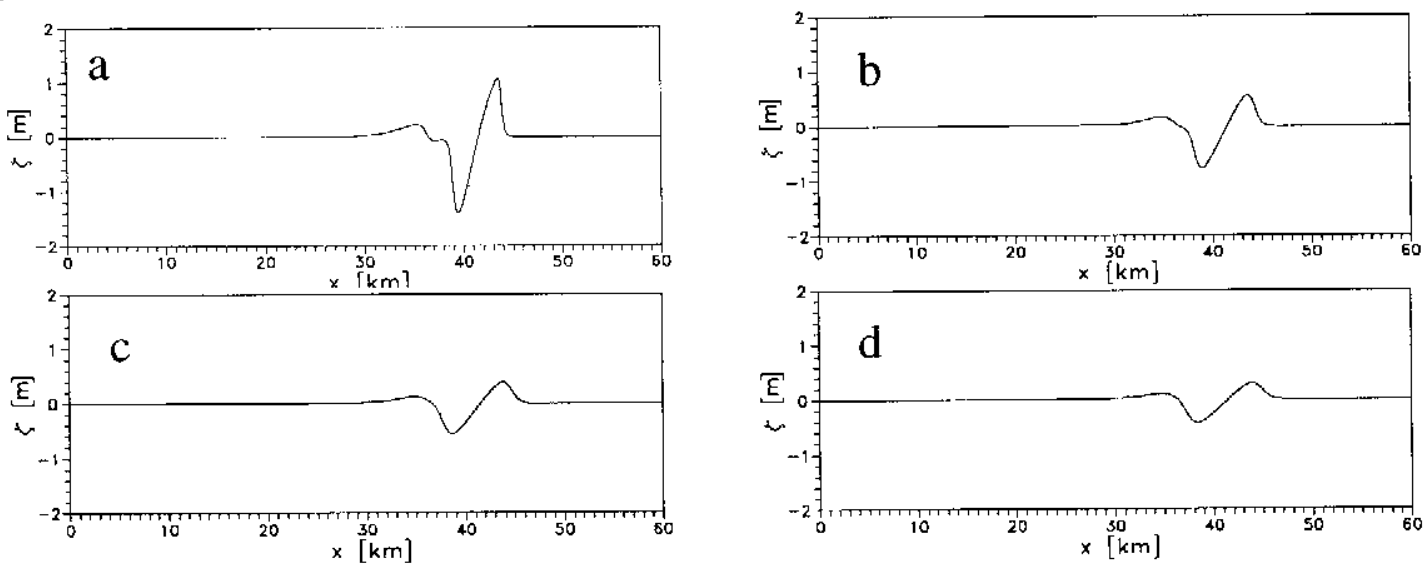

Fig. 5. A: Tsunami evolution for Exp. II, $92 \mathrm{~min}$ after $t_{m}$ but always evolving according to $(1,2)$. The wave is located in sector c. B: Axial sections of the tsunami for Exp. II at $t_{m}$ (a) and after 23 (b), 46 (c) and 69 (d) min. In all cases the wave is located in sector $\mathrm{C}$. 
A
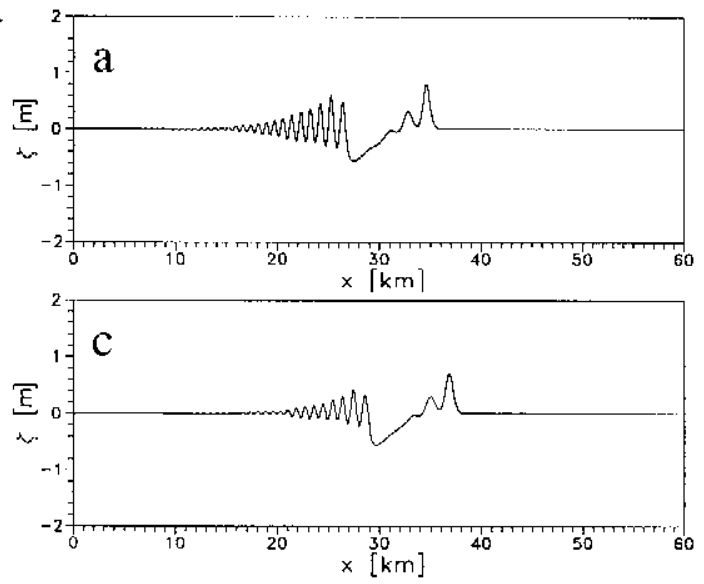

B

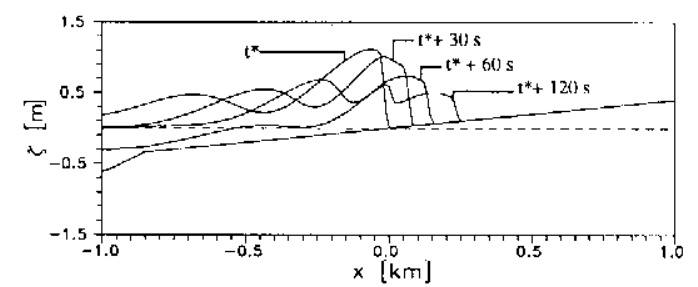

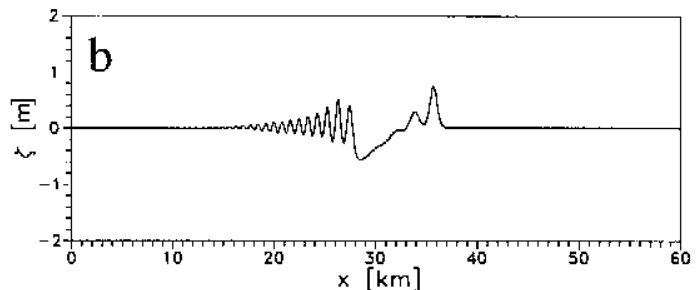

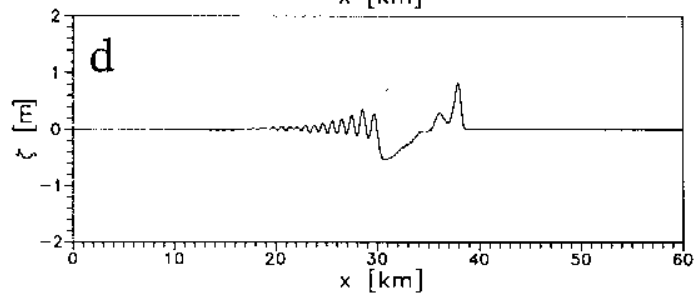

C

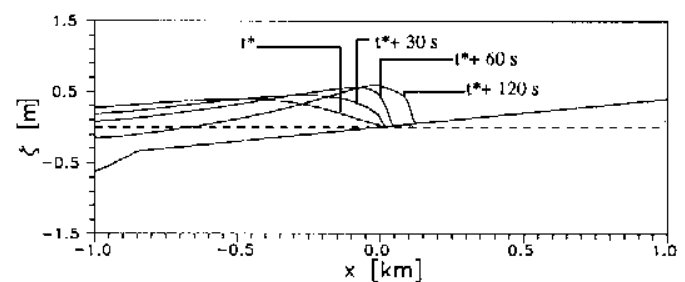

Fig. 6. A: Axial sections of the tsunami for Exp. I, 50 (a), 100 (b), 150 (c) and 200 (d) s after the coupling between the dispersive model (3) and the run-up model $(1,2)$. In all cases the wave is located in the sloping sector d. Axial sections of the tsunami for Exp. I (B) and for Exp. II (C) in the final part of the sloping sector d. At $t=t^{*}$ the first landpoint has just been flooded.
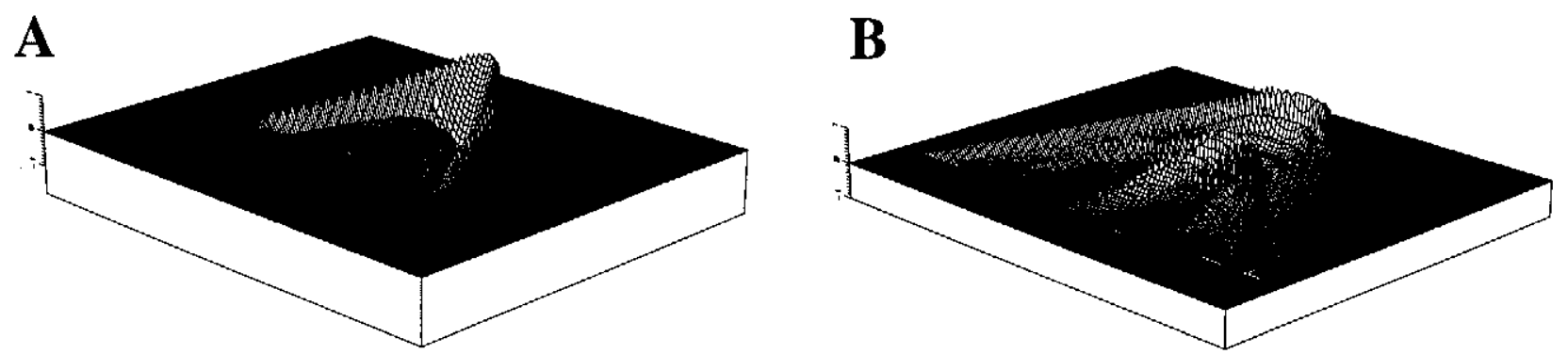

C
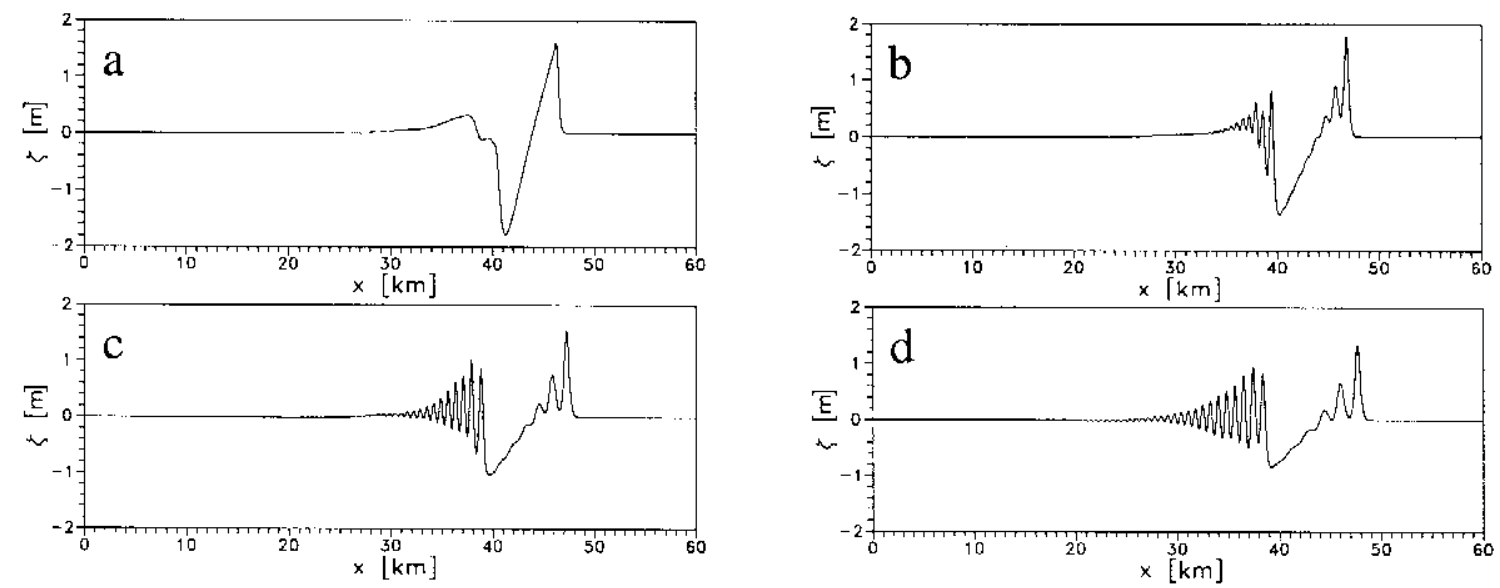

Fig. 7. Tsunami evolution for Exp. III at $t=t_{m}$ (A) and $92 \mathrm{~min}$ (B) after the coupling with the non-hydrostatic model (3). The wave is located in sector c. C: Axial sections of the tsunami for Exp. III at the matching time $t_{m}$ (a) and after 23 (b), 46 (c) and 69 (d) min. In all cases the wave is located in sector $\mathrm{c}$. 

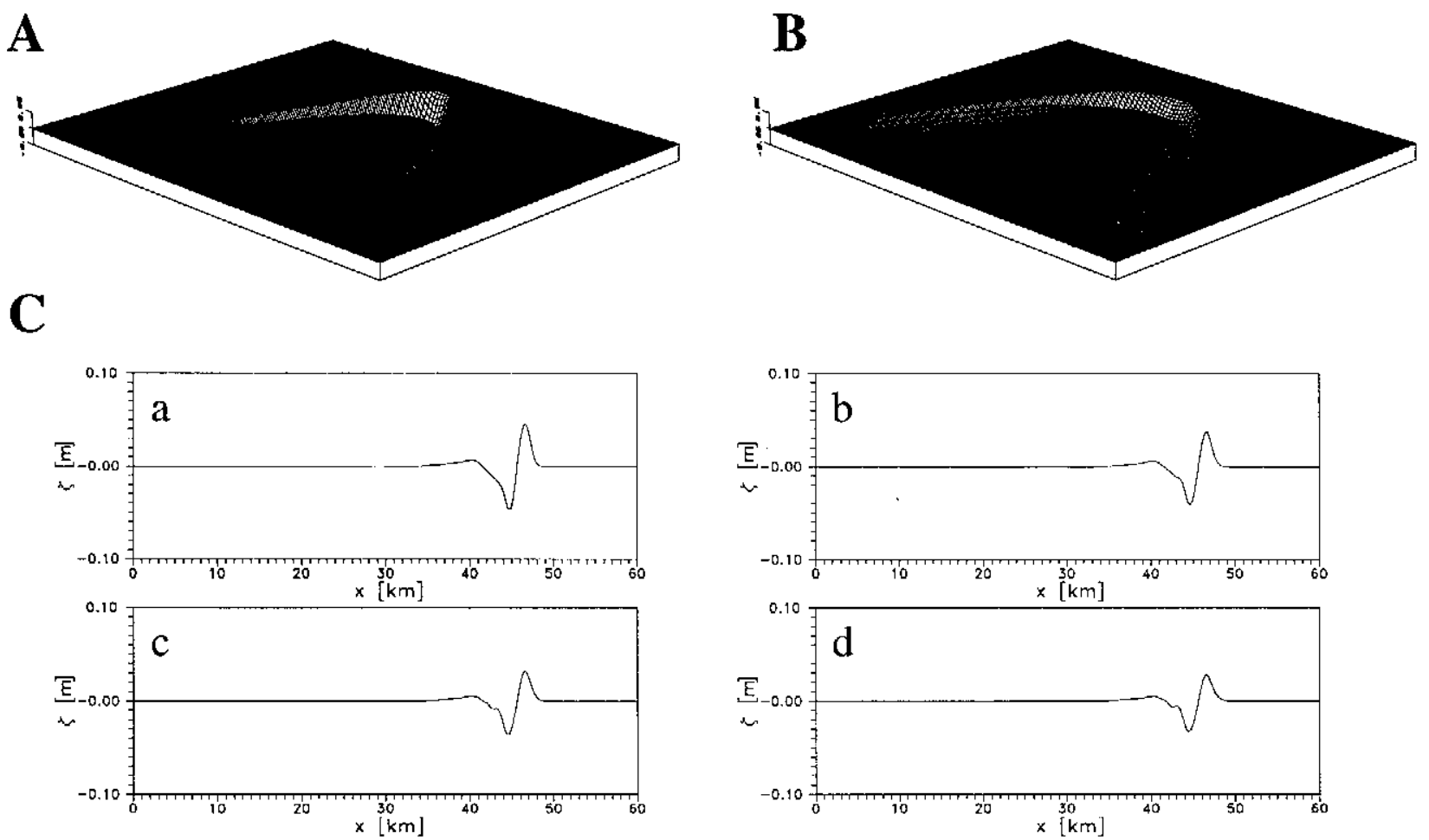

Fig. 8. Tsunami evolution for Exp. IV at $t=t_{m}$ (A) and $92 \mathrm{~min}$ (B) after the coupling with the non-hydrostatic model (3). The wave is located in sector c. C: Axial sections of the tsunami for Exp. IV at the matching time $t_{m}$ (a) and after 23 (b), 46 (c) and 69 (d) min. In all cases the wave is located in sector $\mathrm{c}$.

This evolution is similar to that corresponding to a larger initial slide volume $\left(\mathrm{V}=1.3 \times 10^{8} \mathrm{~m}^{3}\right.$, Exp. VI) but without lateral constrictions (Fig. 11). It has to be stressed that, duc to the presence of the fjord and hence to the reflection thus induced which attenuates the negative part of the wave, the corresponding far-field evolution shows a very weak dispersive tail (Fig. 10C).

\section{Conclusions}

In this paper a process study is presented showing that during the propagation of relatively small energy tsunamis such as mudslide-induced ones, phase dispersion effects can develop and produce solitary waves. This usually happens when the tsunami waves, approaching a coastal region, transit into shallower water. A drastic increase in the Ursell number is then experienced and, conscquently, the rapid development of solitary waves is observed. On the other hand for this kind of coastal tunamis dispersive effects are generally negligible in open occan propagation.

The present process study was based on a nonlinear nested model in which the tsunami generation and run-up were described by the hydrostatic shallow water equations, while the propagation over the far-field shelf region was described by means of the nonlinear and dispersive KP equation. Appropriate matching procedures were developed in order to couple smoothly very different models such as those mentioned above.

It was shown that in some realistic cases nonlinear dispersive tsunami waves can emerge as a result of the evolution of a mudslide-induced initial disturbance. In this case a merely hydrostatic description would lead to erroneous conclusions about wave evolution. We analysed this particulary in connection with run-up estimates and found that, indeed, neglecting phase dispersion can lead to underestimate notably the coastal run-up.

Acknowledgement. We would like to thank Peter Brandt for helpful discussions. This research was partially supported by a „40\%" M.U.R.S.T. project of Italy.

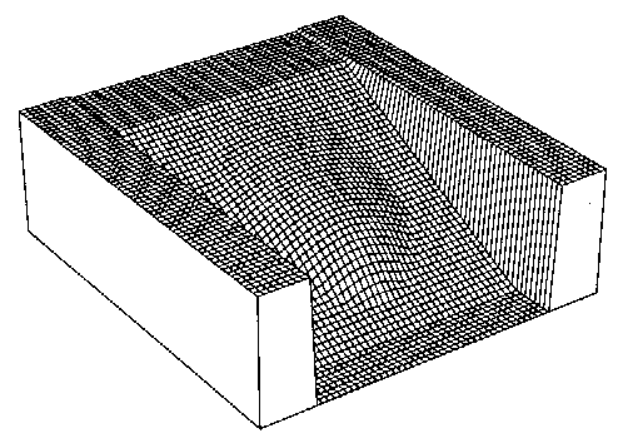

Fig. 9. The topography used in sector a for Exp. V. 

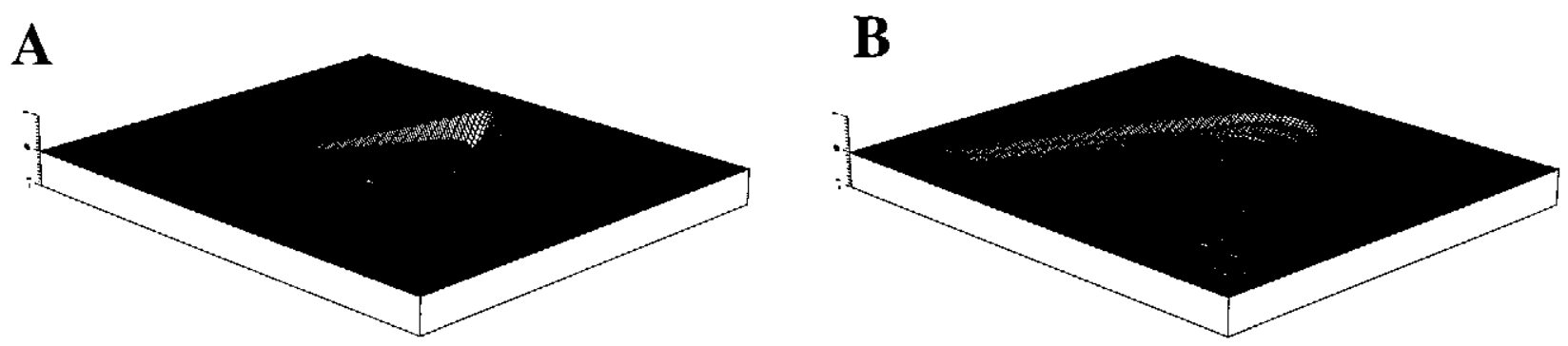

C
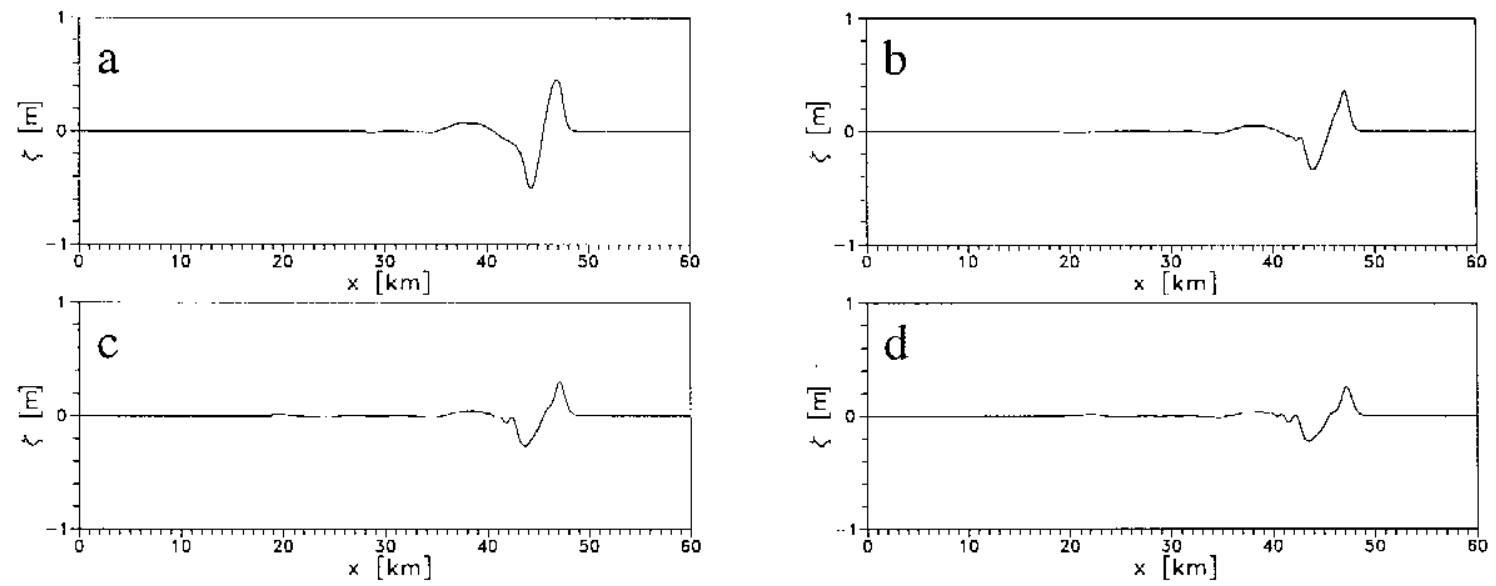

Fig. 10. Tsunami evolution for Exp. $\mathrm{V}$ at $t=t_{m}$ (A) and $92 \mathrm{~min}(\mathrm{~B})$ after the coupling with the non-hydrostatic model (3). The wave is located in sector c. C: Axial sections of the tsunami for Exp. V at the matching time $t_{m}$ (a) and after 23 (b), 46 (c) and 69 (d) min. In all cases the wave is located in sector c.
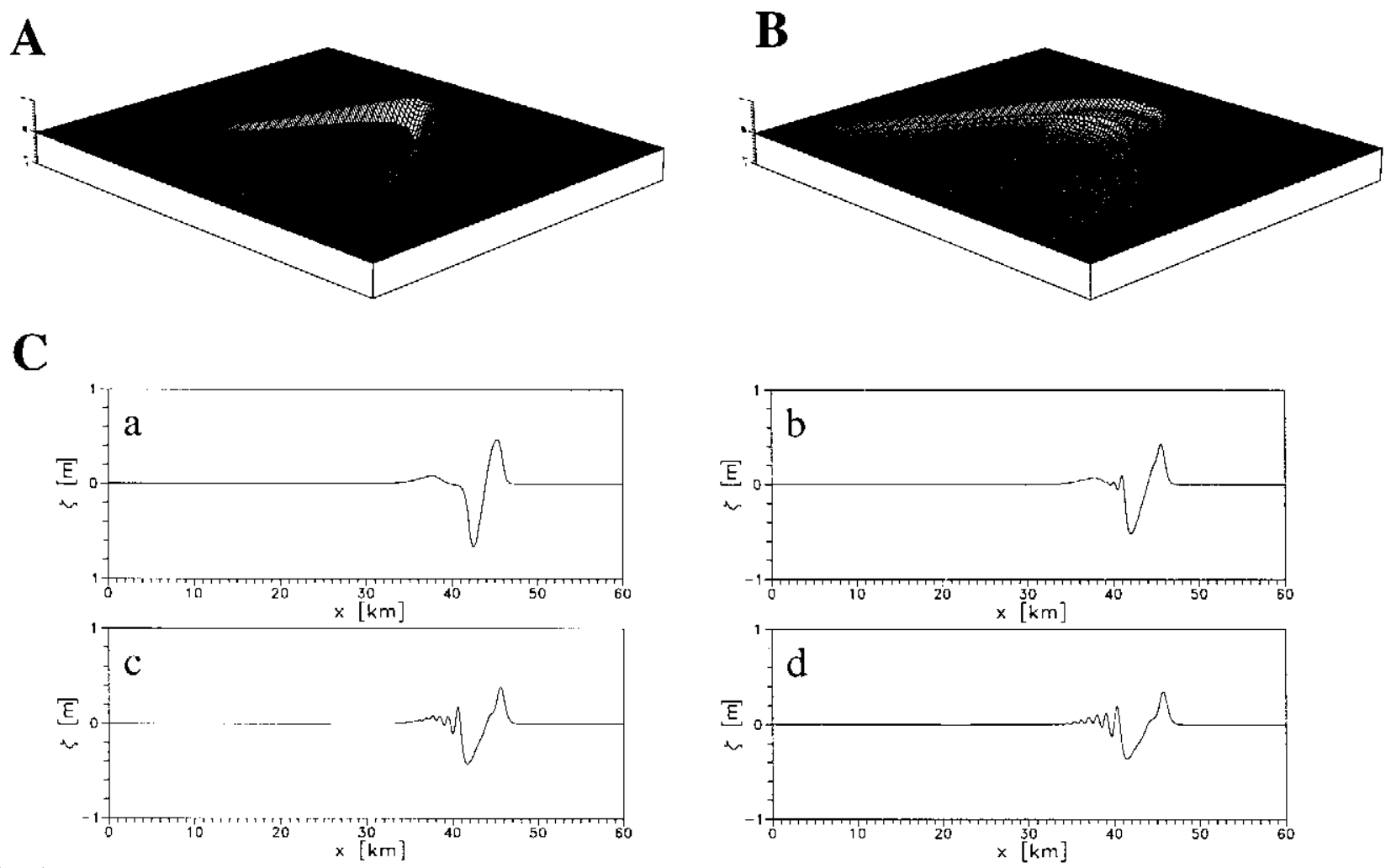

Fig. 11. Tsunami evolution for Exp. VI at $t=t_{m}$ (A) and $92 \mathrm{~min}$ (B) after the coupling with the non-hydrostatic model (3). The wave is located in sector $c$. C: Axial sections of the tsunami for Exp. VI at the matching time $t_{m}$ (a) and after 23 (b), 46 (c) and 69 (d) min. In all cases the wave is located sector $\mathrm{c}$. 


\section{References}

Backhaus, J. O., 1976. Zur Hydrodynamik im Flachwassergebiet, Ein numerisches Modell. Dt. Hydrogr. Z, 29, 222-238.

Hammak, J. L., 1973. A note on tsunamis: their generation and propagation in an ocean of uniform depth. J. Fluid Mech., 60, 769-799.

Hammack, J.L. and H. Segur, 1974. The Korteweg-de Vries equation and water waves. Part 2. Comparison with experiments. J. Fluid Mech., 65, 289-314.

Jiang, L. and P. H. LeBlond, 1992. The coupling of a submarine slide and the surface waves which it generates. J. Geophys. Res., 97, 12731-12744. Jiang, L. and P. H. LeBlond, 1993. Numcrical modeling of an underwater Bingham plastic mudslide and the waves which it generates. J. Geophys. Res., 98, 10303-10317.

Jiang, L. and P. H. LeBlond, 1994. Three dimensional modeling of tsunami generation due to a submarine mudslide. J. Phys. Oceanogr., 24, 559-572.

Jungclaus, J. H. and J. O. Backhaus, 1994. Application of a transient reduced gravity plume model to the Denmark Strait overflow. $J$. Geophys. Res., 99, 12375-12396.

Kadomtsev, B.B. and V.I. Petviashvili, 1970. On the stability of solitary waves in weakly dispersing media. Soviet Phys. Dokl., 15, 539-541.

Kanamori, H. and M. Kikuchi, 1993. The 1992 Nicuragua earthquakc: a slow tsunami-earthquake associated with subducted sediments. Nature, 361, 714-716.

Murty, T. S., 1979. Submarinc slide-generated water waves in Kitimat Inlet, British Columbia. J. Geophys. Res., 84, 7777-7779.

Pierini, S., 1986. Solitons in a channel emerging from a three-dimensional initial wave. Nuovo Cimento, 9C, 1045-1061.

Pierini, S., 1989. A model for the Alboran Sea internal solitary waves. $J$. Phys. Oceanogr., 19, 755-772.

Rubino, A., 1994. Anregung und Ausbreitung von Tsunami-Wellen, die durch untermeerische Erdrutsche verursacht werden. Ph. D. Thesis, Hamburg University, 97 pp.

Sabatier, P. C., 1986. Formation of waves by ground motion. In "Encyclopedia of Fluid Mechanics", 723-759, Gulf Publishing Company.

Satake, K. J., J. Bourgeois, K. Abe, Y. Tsuji, F. Imamura, Y. lio, H. Katao, E. Noguera and F. Estrada, 1993. Tsunami field survcy on the 1992 Nicaragua earthquake. Eos Trans., $A G U, 74,145$.

Tuck, E. and L. S. Hwang, 1972. Long wave generation on a sloping beach. J. Fluid. Mech., 5I, 449-461.

Ursell, F., 1953. The long-wave paradox in the theory of gravity waves. Proc: Cambridge Phyl. Soc, 49, 685-694. 